\title{
Communication \\ Contextualising evidence based medicine in determining the key root for translational effectiveness for chronic disease self-management and heart failure
}

\author{
Pupalan Iyngkaran ${ }^{1,2, *}$, Fahad Hanna $^{3}$, John D Horowitz ${ }^{4}$, Maximilian P. de Courten ${ }^{1}$ \\ ${ }^{1}$ Mitchell Institute for Education and Health Policy, Victoria University, 3000 Melbourne, Australia \\ ${ }^{2}$ School of Medicine Sydney, Werribee Mercy Sub School, University of Notre Dame, 3030 Victoria, Australia \\ ${ }^{3}$ Department of Public Health, Torrens University, 3000 Melbourne, Australia \\ ${ }^{4}$ Basil Hetzel Institute, University of Adelaide, 5011 Woodville South, South Australia, Australia \\ *Correspondence: pupalan.iyngkaran@live.vu.edu.au (Pupalan Iyngkaran) \\ Academic Editor: Vincenzo Lionetti \\ Submitted: 19 October 2021 Revised: 9 November 2021 Accepted: 16 November 2021 Published: 19 January 2022
}

\begin{abstract}
Background: Congestive heart failure (CHF) management has proven devastating on morbidity, mortality, quality of life and also costly to health systems. Therapeutics for CHF have advanced and benefited greatly due to large multicentre randomised controlled trials and the evidence obtained from them. Management for chronic diseases and nonpharmaceutical therapies such as chronic disease selfmanagement has lagged, and for CHF the evidence base has even been questioned. Methods: Perspective and non systematic mini review. Conclusions: Advancing translational research standards is important to achieve optimal cost effectiveness. Importantly is understanding evidence generation in medicine, identifying the primary roots for management and its translation.
\end{abstract}

Keywords: Clinical translation; Congestive heart failure; Evidence based medicine; Guidelines; Self-management

\section{Introduction}

Research in medicine is conducted with multiple goals. The overarching goal is optimised patient outcomes at maximum cost effectiveness. The evidence generated has to take into account confounding variables to limit biases when results are interpreted. Similarly, during clinical translation, variables factored include patients, health practitioners and health systems. Poorly factored are the long timeframes from concept to product, its inherent research costs and also translational pressures particularly cost effectiveness, in real-world clinical settings. Clinical translational is the entire process, including costs, that sees observation, experiments and evidence generated reach the bedside (Table 1). Evidence-based medicine (EBM) popularised in the 1990's is credited with achieving the process that helped generate gold-standard evidence.

Among the specialties to have benefited significantly has been cardiology. This is best highlighted by looking at the current chronic heart failure (CHF) guidelines (20132021) from leading the cardiac societies [1-3]. Noticeable gaps however exist, as the guideline authors themselves suggest, "Despite the objective evidence compiled by the writing committee on the basis of hundreds of clinical trials, there are huge gaps in our knowledge base about many fundamental aspects of CHF care [3]". Some of the main points raised were:

(1) Gaps in knowledge/evidence-cited examples include Heart Failure with preserved Ejection Fraction (HFpEF) beyond blood pressure control, cardiorenal syn- dromes, biomarkers in optimization of therapies and the benefits of sodium restriction.

(2) External validity and translation-

"...the majority of the clinical trials that inform guideline derived medical therapy (GDMT) were designed around the primary endpoint of mortality, so that there is less certainty about the impact of therapies on the healthrelated quality of life (HRQOL) of patients".

"...high prevalence of comorbidities and multiple chronic conditions, but most guidelines are developed for patients with a single disease...Most randomized controlled trials (RCTs) in HF specifically excluded patients with significant other comorbidities from enrollment, thus limiting our ability to generalize our recommendations to many realworld patients ... clinician must, as always, practice the art of using the best of the guideline recommendations as they apply to a specific patient".

(3) Future studies-will continue to focus on disease modifying treatments. Importantly process of care research targeting hospital to home and community care as well as primary prevention will likely have increasing emphasis.

Chronic disease self-management (CDSM) programs for CHF have been tasked with improving translational outcomes, but in itself also faces challenges in evidence translation. The evidence that HF is epidemic is not disputed: lifetime risk of $20 \%$ for ages above 40 years, 650,000 cases yearly, a prevalence of 5.1 million, and cases projected to rise with an aging population and among ethnic minorities [4-7]. The absolute mortality rates, unlike ischemic and 
Table 1. Common Terminology.

(1) Observation: Research, and clinical pathways have a well-defined and governed process that serves a healthy proportion of anycommunity.

(2) Evidence: Several gaps exist including, validity and applicability for a patient cohort well outside the trial cohort characteristics; and cost-effectiveness within a jurisdiction and the system I entirety. While translation of discoveries benefits the population studied, it may raise questions within the wider population.

(3) Clinical Translation: is research that leads to better understanding of disease, and/or development of new diagnostic tests or treatments.

(4) Innovation: Health Practitioners should understand the research framework and engage in continuous professional development as minimal governance and explore new directions as new observations present.

(5) Health Services: should invest in systems and infrastructure that filter relevant observations or research questions that inform cost-effectiveness.

(6) Governance: Health clusters could be the minimum accountable boundary for cost-effectiveness governance.

(7) Generalizability: degree to which the results of a research study reflect what the results would be "in the real world", with another sample of participants or with the variables operationalized in other ways.

(8) Clinical applicability: the extent to which the users can apply a recommendation in practice.

(9) Standardisation: Framework for research translations will help bridge silos between health administrators and clinical practitioners.

(10) Clinical Translation: is research that leads to better understanding of disease, and/or development of new diagnostic tests or treatments.

(11) Implementation: integrated concept that links research and practice to accelerate the development and delivery of public health approaches. Implementation research involves the creation and application of knowledge to improve the implementation of health policies, programs, and practices.

other cardiovascular conditions, have remain unchanged at $50 \%$ at 5 years from initial diagnosis [8]; furthermore, costs are high along with high rates of recurrent hospitalisation at $25 \%$ within 30 days of discharge [9], alongside reduced HRQOL [10,11]. At least 50\% have one, and $>20 \%$ have more than one comorbid conditions, which also results in many being excluded from clinical trials [3,9].

Prior to moving forward, we acknowledge the themes argued on EBM, and clarify there continue to be positive changes, e.g., in newer guidelines [12] and clinical trial enrolment, e.g., representative patient population with comorbidities as seen in Emperor Preserved for SGLT2 inhibitor [13]. In this perspectives, we thus explore the subject of CDSM and HF from several angles: firstly, the historical context of evidence-based medicine; secondly, the paradigm that is evolving for translational sciences and its standardisation.

\section{Perspective on medical evidence}

\subsection{The rise of the philosophy of scientific experimentation}

The Sixteen and Seventeenth centuries saw a rise in experimental sciences with mathematical methodology better known as Baconian or mechanisation worldviews. In time this evolved to more radical experimentation and scientific thought. However, Kuhn argues it was only at the second half of the nineteenth century that a systematic interaction and merging of experimental and mathematical paradigms took shape [14]. Following this period medi- cal sciences had unprecedented exposure to a modernising, technologically advancing world and with it a profusion of information. The goal across era's however remains unchanged: to provide medical care, improve patient's quality of life and health outcomes; and the framework for this is consensus governed evidence standards, consensus interpretation of evidence and clinical translation as guided by jurisdiction defined cost-effectiveness. Much has been achieved in standardised processes of care, frameworks, and guidelines. Krumholtz's taxonomies of disease management [3], is an important framework, that highlight eight domains from which to prioritise disease management discussions. To populate these domains, we utilise EBM to ask what actually works, what is still working and what do we need to better understand in medicine?

The hallmark of modern medicine was highlighted in the early 1990's, that saw the EBM movement and important initiatives like the Cochrane Collaboration, reinvigorate objectivity as a core pillar in evidence and its translation in medical decision taking and practice. Predominately it was the larger studies, that generated evidence, and coincidentally this weighted toward pharmaceuticals and devicebased interventions. Subsequently this process developed into an industry where some important themes were becoming prominent: firstly were questions of biases, and conflicts of interest in the investigative process; secondly, translational gaps were developing particularly in the applicability and generalisability outside clinical trial populations; thirdly, opinions were being voiced on constraints on 
freedoms to make clinical judgements and practise the artistic side in clinical practice(lay terms for these include PubTest or common sense). These terms relate to clinicians' experience and reasoning, or clinical judgement when executing solutions at the bedside; fourthly, an extension from the third point were the development of silos between aspects of the EBM systems and grey areas in clinical practice. Examples of these are seen daily with off label use of medications and in individualising treatments where physicians have to account for certain conditions being factored the potential benefits or side-effects given different patient characeristics [15-17].

There is thus an important philosophical question when researching medical solutions, i.e., what is being taught, how do we interpret this within a given context and how should we explore the problem, should there be variations from the taught observation? EBM has taught us that reasoning, process, controlled observation, and experimentation are vital for accurate findings. However, there is a second part which is less well enforced, this importantly, is what do we need to learn about the environment and populations that the findings are applied to? Beyond clinical translation of evidence, it is possible to encounter resistance from practitioners whose personal experiences and patient population differ significantly from which evidence is generated. In therapeutics this can result from rigidity in interpreting trial findings. As an example is the observed drug effect a specific effect or one shared within drugs of the same class. This can be important when drugs within a class have extra class benefits that may have benefits for populations with comorbidities [18]. Such clinical scenarios will face many professionals. As such all-health protagonists are also teachers and students in this process, where the unifying standard is one of governance. The current structures to gain, interpret and standardise evidence are quite robust; however it remains unclear whether consensus guidelines are a framework or guide and where the margins for flexibility are? In guidelines, evidence is classified as Class 1-III (size of treatment effect), Level A-C (estimate of certainty or precision of treatment effect) and T1-3 (translation level from bench to community) [3]. The artistry of clinical practice will probably be a lot harder to compartmentalise and could be a topic for future discussions.

\subsection{Traditional research paradigms}

\subsubsection{Foundations}

The framework for Western medicine is built on the paradigm of sequence; for example, there are disease and management taxonomies, which branch into clinical domains, which are then populated with variables that can be actioned. The framework for evidence has a similar taxonomy which are measures of a clinical standard and appropriately called performance measures or indicators. This framework for evidence is overseen by additional standardisation framework which delivers consensus on the pa- rameters for the observations. Paradigms change when the parameters within any framework shift beyond the current boundaries to form a new framework. Cardiology research thus has two essential ingredients as its unchanging paradigms, firstly observation and its description within these standardised measures; and secondly governance-ongovernance which is monitoring of all steps in all the processes. In practice health professionals have to ensure the standard of care that is delivered is evidenced and uptodate. The expectations on experience, to bridge tough clinical problem areas will always have debate on its latitude. It must also be highlighted that frameworks are set by International Consensus Groups, National bodies such as Speciality Colleges, and are enforced by approved institutions like hospitals.

Research starts with the intention of establishing an answer, for a defined question through a systematic process [19]. Living systems maintain homeostasis, through a dynamic environment, thus evidence has an applicability and a shelf-life. Whether through incidental observations or scheduled governance audits, new findings may arise, some of which requires action. Th journey to address these observations can occur via literature reviews and when data is pooled, meta-analysis or systematic reviews can generate new perspectives. If novel data is required, observations, case and cohort studies are conducted. Experiments can also be designed on animals and humans. When an understanding takes shape, the findings can be consolidated by testing the hypotheses in controlled studies, where bias is accounted for. The process from initial observation to clinical trials can traditionally take years and require large funds (Fig. 1). Very importantly, it is assumed that the findings are clinically and universally translatable.

EBM has set a high bar particularly with the concepts of Level of evidence and Strength of Evidence. These pillars have several ramifications: firstly, evidence can be generated at many phases of research however to establish confidence in the finding requires more rigor; secondly, as phase-4 (post-trial) studies are not mandatory or even feasible across many health systems, clinical translation has required a degree of subjectivity. It is important for us to consolidate on the important achievements via the EBM process. Equally we must continuously explore avenues for greater types of evidence to be more easily translatable and also improve the population diversity participating in gold standard large multicenter trials.

Presently there are no guidelines for these. These points are quite relevant for the example we explore in this perspective. CDSM and HF evidence has been downgraded and there appears little coherence as to what CDSM could look like in daily practice $[2,20]$. Our team have published and argued that as the HF guidelines are established, the question of CDSM is thus one of helping achieve GDMT. In addition, CDSM programs do not independently need to influence all major cardiovascular event rates (MACE). In 


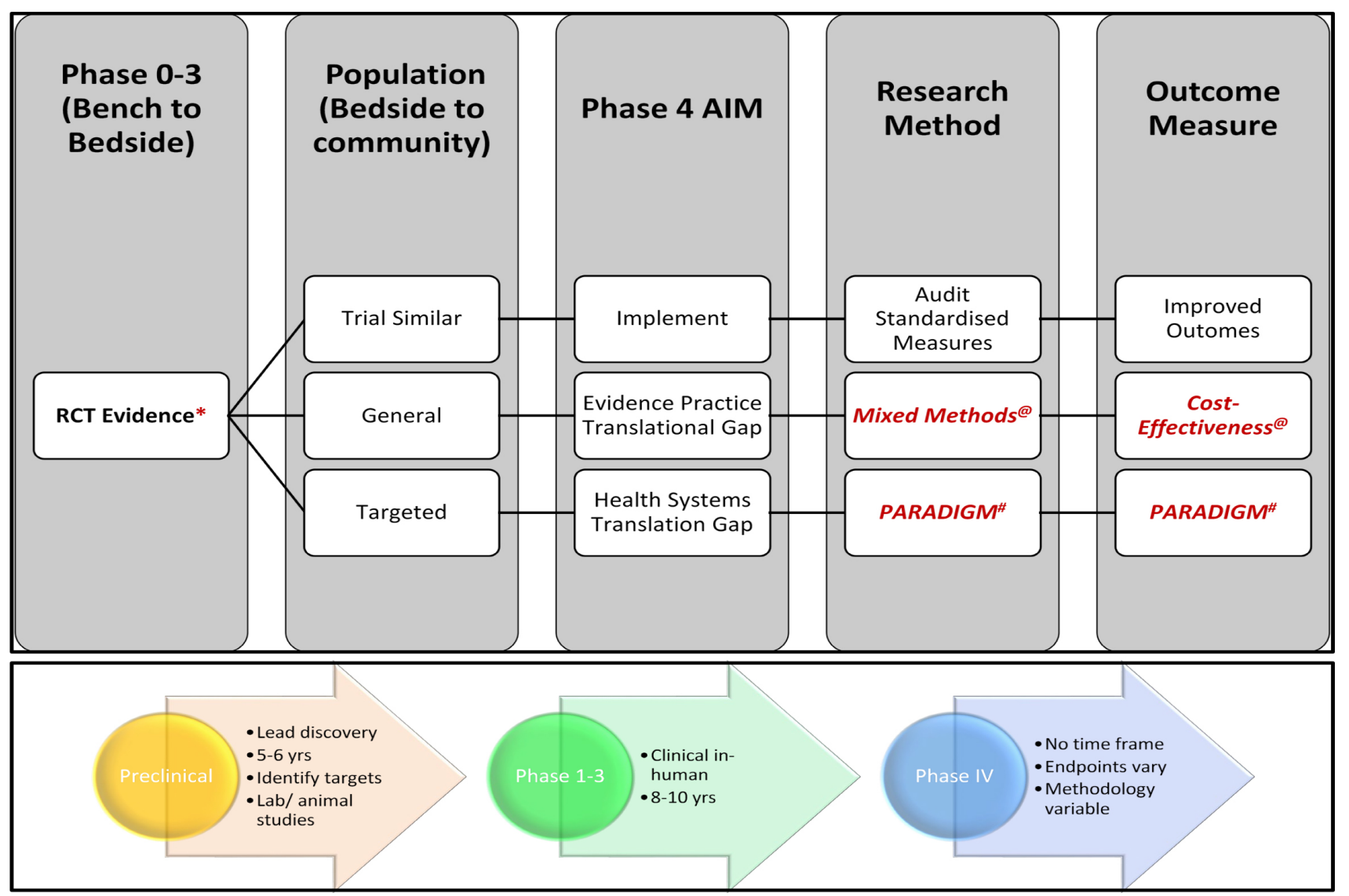

Fig. 1. Flow diagram of Evidence and Paradigm Gaps. Research generated in phase 0 and validated in phase 3 constitutes gold standard evidence. Phase-4 clinical translation can be straightforward or require greater investment. There are gaps in translating evidence at a population level that can range from minor to significant. These areas may form the basis for future consensus. * Gold standard evidence achieved. @ Established evidence will face translational issues; greater consensus needed on characteristics of this new evidence needed and outcome measures that will support clinical translation. \# New paradigm may be needed to see greater translation for heterogenous populations.

doing so, we are then able to introduce a process of care tool that enhances uptake of optimal care (GDMT), at a lower cost and in doing so deliver the proven outcomes.

\subsubsection{What have we learned?}

We have learnt a lot and come very far. In taking time to see if the gains have been uniform, we can learn what has been left behind. Registries are the most recognized observational tool where the parameters or performance measures extracted determines where and what the registry informs. RCT establish gold standard proof of hypotheses (efficacy) and are the foundations of EBM. They are not however efficient at establishing effectiveness and clinical translation aims. RCT governance process of itself has boundaries. In regards to evidence-translation we can thus explore this into two parts (Table 1: firstly, what is the generalisability of the evidence in the community? and what is the applicability of the evidence in individual clinical practice [21-28]? Some relevant points on this:

(1) As governance is greater, internal validity con- trol of bias increases but external validity (applicability and generalisability) diminishes.

(2) External validity is a matter of individual judgement. While some higher risk groups are increasingly highlighted in guidelines, actual solutions are lacking.

(3) Industry has an important role in medical innovation, and they must be supported within the frameworks created in all research aspects.

(4) Greater control and leverage must be exerted, outside industry, on post-translational works to broaden the evidence base.

(5) The existing trial infrastructure could be utilised to achieve this goal which then informs subsequent health economic evaluation.

In reality however, both groups, those represented in trials or those that are not, can be left behind in real world translation. For routine patients this is best demonstrated by OPTIMIZE-HF trial where performance measures, not trial protocol, improves MACE and QOL [29]; similarly, groups not conventionally represented in trials can suffer adverse 


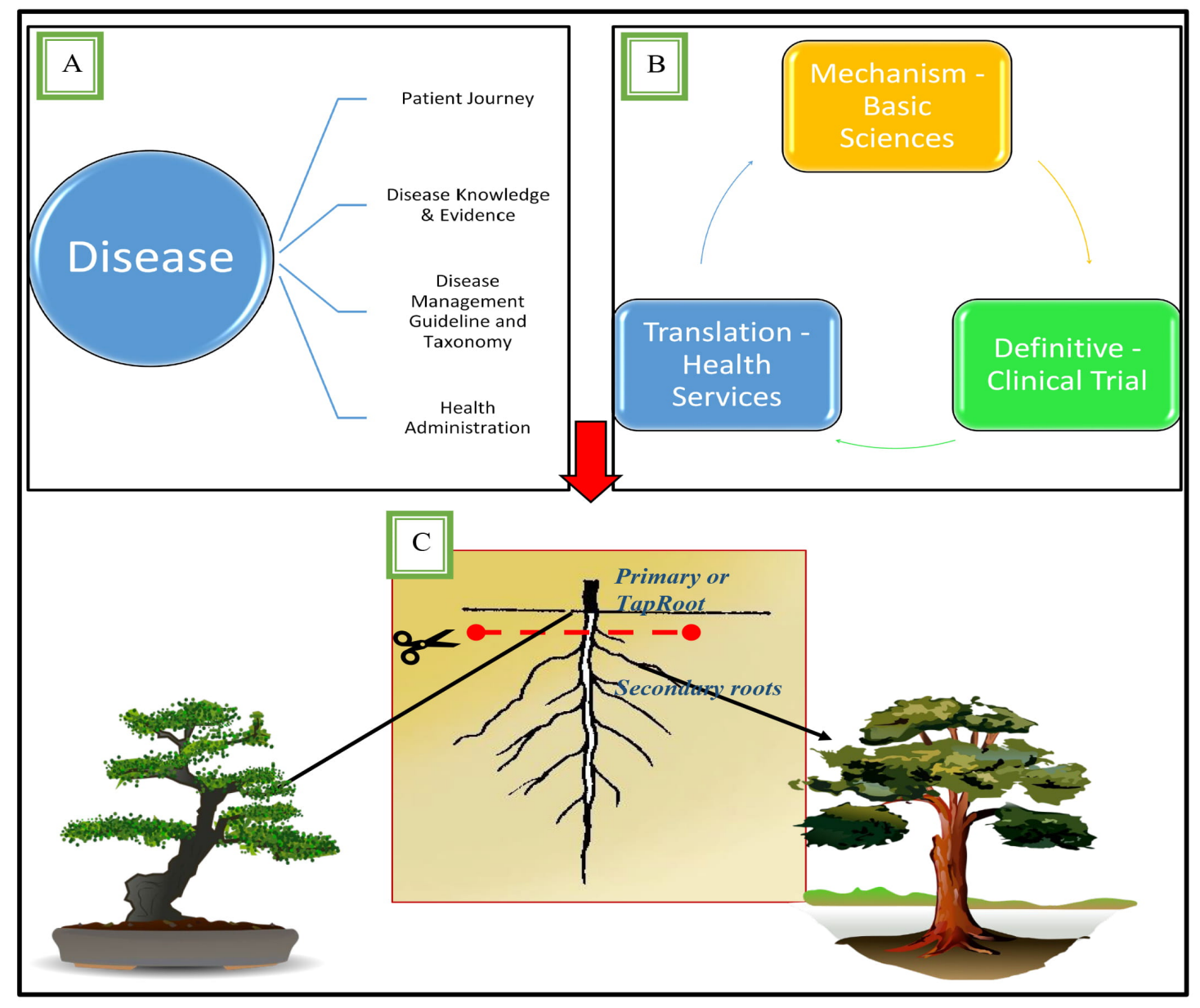

Fig. 2. Taproot concept and defining key research strategy. Diseases like all systems have historical journeys and future trajectories. (A) Assuming a critical juncture for evidence in disease management exists, thought processes for population level clinical translation should aim to identify the primary/Taproot or key performance factor for relevant health clusters that provide that jurisdiction greatest cost-effectiveness. (B) Evidence based medicine is a process with levels and grades of evidence, la being the strongest. This is often best obtained through findings from phase 0 (basic mechanistic) to phase III (definitive and reproducible randomised trials). Phase IV (Translational and health services) research may require mixed methods, e.g., qualitative research which is more difficult to grade. (C) In the primary or Taproot system, the growth of the future tree is based on health of the taproot that gives rise to secondary and smaller root systems. If the trees are equated to the disease burden, and roots the growth pathways, targeting the taproot is of importance as it either results in a pot sized tree (Bonsai) or fully grown oak tree. Thus the hypothesis, question and methodology required is vital to determine how the evidence is interpreted and the ability to dedicate resource towards that cost-effective solution.

outcomes, not developing in the trial, as seen with lisinopril cerebrovascular events, angiotensin converting enzyme monotherapy efficacy in African Americans or statin dosing in Asians [30,31].

While RCT driven evidence has unequivocally increased population cardiovascular health, it will never be possible to find evidence at an individual level. The focus should be on understanding what constitutes a significant difference in an observation following implementation of trial findings, that warrant further attention. This link between evidence generation and research translation or $E v$ idence Translation, is inherently linked with observation. What is different however is there must be better mechanisms for finding both evidence and translational solutions, so as not to delay the implementation of strong RCT findings.

It is thus important we clarify that while guidelines are the best reference for current evidence, however they are not performance measures or barometers of real world clinical practice [32-36]. They can be considered an instruction manual but in using them we must always be cognizant the there is a shade of grey when clinical judgement is required. Translational or Implementation sciences are thus a governance process linked to guidelines, are poorly understood and can be poorly utilised. An important consideration for this time is the interaction of quantitative reasoning and methodological perfection (EBM) and its application that leaves lesser and lesser room for qualitative reasoning 
or clinical judgement. As a reflection we could pause and analyse what are acceptable gains and losses for the merging of scientific reasoning with EBM and evolving realities clinicians are facing?

\subsubsection{What can we do better?}

A key deficiency in EBM is the cost and time to attain Gold Standard Evidence, that make up guidelines. An important finding can be also defined as rate limiting factor, key determinants or in another analogy the Taproot (Fig. 2). Here an intervention at a key point in the tree's development can drastically alter its size. It does make sense that some questions will require completely novel findings using bench to bedside basic science and drug discovery. However, in cost-effectiveness many of the pieces are known, and what may be required are new arrangements. The taproot in this case may require a qualitative approach in a smaller subset to establish gold standard evidence. Thus, to determine the key determinants, perhaps for a defined region to achieve a good translational outcome, could require different standards (Fig. 3). Using a different example, question have been raised of perioperative myocardial cell injury, anesthesia and findings for larger trials of cardioprotective agents. Clearly in this scenario, this translational issue is one that requires further pathophysiological understanding at the basic sciences level. In the 1980's concern of volatile anesthesia and silent ischemic burden were not backed by trial findings where $70 \%$ of patients had intracardiac protective agent. The association between agent toxicity versus cardioprotection could not be corroborated [37]. Publications a decade later support the notion that surgery is safer in the current era however rates of perioperative major adverse cardiovascular events, are still a significant clinical problem [38,39]. While we have raised the argument for mixed method research guidelines for translational research on health services needs within health clusters, we certainly support others exploring the bedside to bench translational guidelines for efficacious phase-IV research for these issues.

\section{Standardising actionable posttranslational evidence-the precipice of a new paradigm?}

The Framingham study from the mid twentieth century largely from observation drew attention to novel CHF phenotypes, e.g., HFrEF and HFpEF and using conventional research methodologies clinical understanding was improved. With the advent of RCT's as a mainstay for evidence, there are increasing observations of translational issues and outcome gaps in populations. There remains a suboptimal process that transitions the findings from lightly controlled clinical trials and attaining the outcomes demonstrated in real world clinical practices and global population level [40-42]. This issue can be explored further by 3 essential questions which highlight some of these deficiencies, on emphasis on post-trial processes:
(1) The RCT is gold standard for evidence, should phase IV research be considered a necessary translation and implementation tool for populations with large differences from trial enrollment criteria?

(2) Many HRQOL, translation and implementation research utilize qualitative and mixed method research which are not classified as gold standard, it is unclear the appropriate metrics to explore to find this post-trial gold standard evidence?

(3) How does one introduce novel observations, if it must fit within the regulated process, for example, a new idea or abstract thoughts may not have preceding references to it?

Fundamentally, when answering the question of CDSM for HF, we must accept that we are on the precipice of a new paradigm. The foundations of these are fundamentally the disconnect between what is actionable; in fact, DeMaria suggested the overreliance on RCT data is causing paralysis in clinical decision making among current trainees [43]. CDSM was a new measure in 2013 HF guidelines, measuring $>3$ elements of education, $>1$ visit over 12-month period, as an outpatient by individual practitioners. This standard was also recommended for internal quality improvement programs and bound to remuneration 3. A review of disease management programs for vulnerable HF patients, Clark et al. found no or little benefit from these programs [2,42-48]. This and other studies went on to shape a downgrade of CDSM evidence in $2021 \mathrm{HF}$ guidelines. This highlights the value in reporting non-pharmacological trials around the CONSORT statement, which considers systems and contextual factors in health systems research [30,49-51]. While there are arguments that all evidence is not readily generalisable and there are gaps in acquiring actionable translational evidence, Nieuwlaat et.al argues that the evidence practice gaps are underuse of proven, overuse of unproven, knowledge, strategy, and structural barriers to reducing evidence practice gaps [31].

CDSM for HF can evolve as the single most important translational tool to implement guideline-based evidence. In the conventional sense, without a paradigm change in thinking, the gold standard evidence is lacking. A combination of funding, inability to deliver trials with significant improvement in MACE and the subject matter that borders cardiovascular sciences to grasp a wholesome interest from the cardiology speciality. Nonetheless what are is on offer is the opportunity to produce good self-managers, that are compliant, who can monitor variables of their chronic illness including changes in disease status and action strategies such as fluid balance and medications. This selfmanaged HF patient would reduce health services resourcing and lead to significant cost savings [52]. Fig. 1 provides a brief outline to further future discussions. In summation, to move forward on this issue and in planning clinical research using CDSM programs for HF, should we consider 


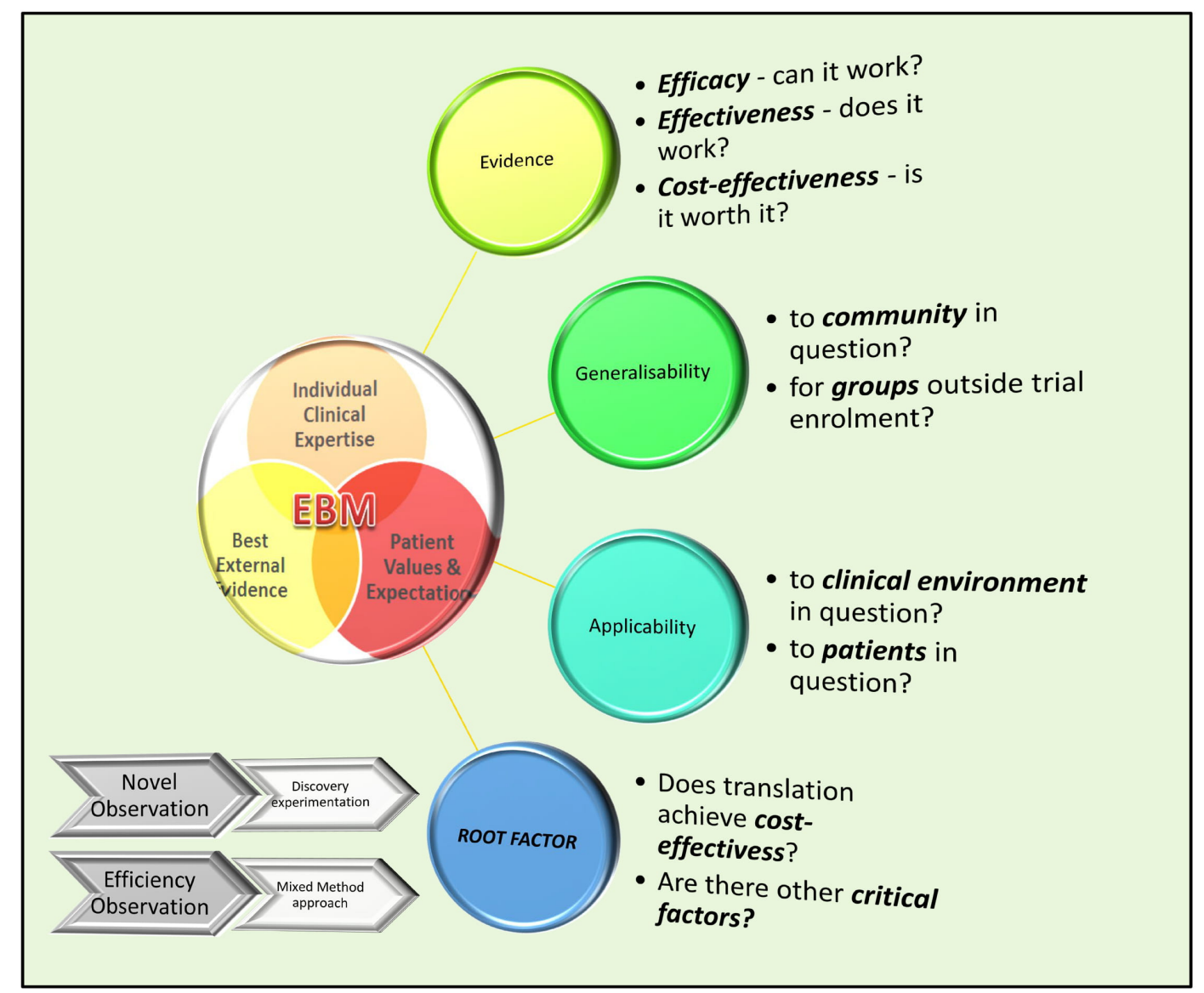

Fig. 3. Making sense of the evidence tree. Translation of good evidence requires exploring the generalisability and applicability of evidence to any defined community. To achieve maximum jurisdiction defined cost-effectiveness any critical barriers must be addressed. The Root Factor is a defining issue, if addressed will help achieve that goal. Phase IV research aims to address this. Mixed method research can be an important means to gather evidence. There remain gaps in classifying and standardising evidence generated with this method for clinical translation (Table 1 provides the definition for the terms used).

that there is sufficient evidence on proven HF treatments thus CDSM is a phase-IV strategy; if so, what are the appropriate research strategy and outcome measures to deliver gold standard and translatable evidence? Are appropriate outcome metrics such as improved uptake, HRQOL and cost-effectiveness sufficient to deliver high level guideline recommendations or is new RCT evidence needed?

\section{Conclusions}

In health system identyfing the key effector or rate determining step could provide a diretion to place critical resources. HF isa journey from the patients perspective, and health systems perspective. In the later a mix of research and evidence, a management classification system and health administration regulation on how care is resourced are important considerations. To effect change in any of these points, depending on the question being researched, quantitative or qualitative research methods can be used. EBM has delivered new benchmarks for practice through a process of securing strong evidence. A gap in this process is effecting translation of this evidence to broader clinical scenarious.An important exmaple is addresing primary issues or as we highlight the anology of a taproot system. Should key factors fall outside the traditional evidence genrating mechanism a long process of generating goldstandard evidence is required. There is potentially a paradigm on the horizon for classification and standardising post-trial or phase-IV research. This may encompass broader research strategies.

\section{Author contributions}

PI-wrote manuscript; MDC, FH, JDH-provided advise on technical aspects. All authors read and edited the manuscript.

\section{Ethics approval and consent to participate}

Not applicable.

\section{Acknowledgment}

Not applicable. 


\section{Funding}

This research received no external funding.

\section{Conflict of interest}

The authors declare no conflict of interest.

\section{References}

[1] Yancy CW, Jessup M, Bozkurt B, Butler J, Casey DE Jr, Colvin MM, et al. 2017 ACC/AHA/HFSA focused update of the 2013 ACCF/AHA guideline for the management of heart failure: a report of the American College of Cardiology/American Heart Association Task Force on Clinical Practice Guidelines and the Heart Failure Society of America. Circulation. 2017; 136: e137161

[2] McDonagh TA, Metra M, Adamo M, Gardner RS, Baumbach A, Böhm M, et al. 2021 ESC Guidelines for the diagnosis and treatment of acute and chronic heart failure. European Society of Cardiology. 2021; 42: 3599-3726.

[3] Yancy CW, Jessup M, Bozkurt B, Butler J, Casey DE Jr, Drazner $\mathrm{MH}$, et al. $2013 \mathrm{ACCF} / \mathrm{AHA}$ guideline for the management of heart failure: a report of the American College of Cardiology Foundation/American Heart Association Task Force on Practice Guidelines. Circulation. 2013; 128: e240-e327.

[4] Go AS, Mozaffarian D, Roger VL, Benjamin EJ, Berry JD, Borden WB, et al. Heart disease and stroke statistics-2013 update: a report from the American Heart Association. Circulation 2013; 127: e6-245.

[5] Curtis LH. Incidence and prevalence of heart failure in elderly persons, 1994-2003. Archives of Internal Medicine. 2008; 168: 418.

[6] Roger VL. Trends in heart failure incidence and survival in a community-based population. Journal of the American Medical Association. 2004; 292: 344.

[7] Bahrami H, Kronmal R, Bluemke DA, Olson J, Shea S, Liu K, et al. Differences in the incidence of congestive heart failure by ethnicity. Archives of Internal Medicine. 2008; 168: 2138.

[8] Loehr LR, Rosamond WD, Chang PP, Folsom AR, Chambless LE. Heart failure incidence and survival (from the atherosclerosis risk in communities study). The American Journal of Cardiology. 2008; 101: 1016-1022.

[9] Iyngkaran P, Liew D, Neil C, Driscoll A, Marwick TH, Hare DL. Moving from heart failure guidelines to clinical practice: gaps contributing to readmissions in patients with multiple comorbidities and older age. Cardiology. 2018; 12: 117954681880935.

[10] Heo S, Doering LV, Widener J, Moser DK. Predictors and effect of physical symptom status on health-related quality of life in patients with heart failure. American Journal of Critical Care. 2008; 17: 124-132.

[11] Lesman-Leegte I, Jaarsma T, Coyne JC, Hillege HL, Van Veldhuisen DJ, Sanderman R. Quality of life and depressive symptoms in the elderly: a comparison between patients with heart failure and age- and gender-matched community controls. Journal of Cardiac Failure. 2009; 15: 17-23.

[12] McDonagh TA, Metra M, Adamo M, Gardner RS, Baumbach A, Böhm M, et al. 2021 ESC guidelines for the diagnosis and treatment of acute and chronic heart failure: developed by the task force for the diagnosis and treatment of acute and chronic heart failure of the European Society of Cardiology (ESC) With the special contribution of the Heart Failure Association (HFA) of the ESC. European Heart Journal. 2021; 42: 3599-3726.

[13] Anker SD, Butler J, Filippatos G, Ferreira JP, Bocchi E, Böhm $\mathrm{M}$, et al. Empagliflozin in heart failure with a preserved ejection fraction. New England Journal of Medicine. 2021; 385: 1451-
1461.

[14] Radder H. The philosophy of scientific experimentation: a review. Automated Experimentation. 2009; 1: 2.

[15] Stone GW, Pocock SJ. Randomized trials, statistics, and clinical inference. Journal of the American College of Cardiology. 2010; 55: 428-431.

[16] Ting HH, Shojania KG, Montori VM, Bradley EH. Quality improvement. Circulation. 2009; 119: 1962-1974.

[17] Geboers H, van der Horst M, Mokkink H, van Montfort P, van den Bosch W, van den Hoogen $\mathrm{H}$, et al. Setting up improvement projects in small scale primary care practices: feasibility of a model for continuous quality improvement. Quality and Safety in Health Care. 1999; 8: 36-42.

[18] Iyngkaran P, Majoni W, Cass A, Sanders P, Ronco C, Brady S, et al. Northern territory perspectives on heart failure with comorbidities - understanding trial validity and exploring collaborative opportunities to broaden the evidence base. Heart, Lung and Circulation. 2015; 24: 536-543.

[19] Geboers H, Mokkink H, Van Montfort P, Van Den Hoogen H, Van Den Bosch W, Grol R. Continuous quality improvement in small general medical practices: the attitudes of general practitioners and other practice staff. International Journal for Quality in Health Care. 2001; 13: 391-397.

[20] Iyngkaran P, R. Toukhsati S, Harris M, Connors C, Kangaharan $\mathrm{N}$, Ilton $\mathrm{M}$, et al. Self managing heart failure in remote australia - translating concepts into clinical practice. Current Cardiology Reviews. 2016; 12: 270-284.

[21] Nieuwlaat R, Schwalm J, Khatib R, Yusuf S. Why are we failing to implement effective therapies in cardiovascular disease? European Heart Journal. 2013; 34: 1262-1269.

[22] Iyngkaran P, Liew D, McDonald P, C. Thomas M, Reid C, Chew $\mathrm{D}$, et al. Phase 4 studies in heart failure - what is done and what is needed? Current Cardiology Reviews. 2016; 12: 216-230.

[23] Geboers H, van der Horst M, Mokkink H, van Montfort P, van den Bosch W, van den Hoogen $\mathrm{H}$, et al. Setting up improvement projects in small scale primary care practices: feasibility of a model for continuous quality improvement. Quality and Safety in Health Care. 1999; 8: 36-42.

[24] Shojania KG, McDonald KM, Wachter RM, Owens DK. Closing the quality gap: a critical analysis of quality improvement strategies. Agency for healthcare research and quality publication No. 04-0051-1. 2004. Available at: http://www.ahrq.gov/downloa ds/pub/evidence/pdf/qualgap1/qualgap1.pdf (Accessed: 6 July 2021).

[25] Iyngkaran P, Liew D, McDonald P, C. Thomas M, Reid C, Chew $\mathrm{D}$, et al. Phase 4 studies in heart failure - what is done and what is needed? Current Cardiology Reviews. 2016; 12: 216-230.

[26] Hlatky MA, Douglas PS, Cook NL, Wells B, Benjamin EJ, Dickersin $\mathrm{K}$, et al. Future directions for cardiovascular disease comparative effectiveness research. Journal of the American College of Cardiology. 2012; 60: 569-580.

[27] Gitt AK, Bueno H, Danchin N, Fox K, Hochadel M, Kearney P, et al. The role of cardiac registries in evidence-based medicine. European Heart Journal. 2010; 31: 525-529.

[28] Grol R, Dalhuijsen J, Thomas S, Veld CI', Rutten G, Mokkink H. Attributes of clinical guidelines that influence use of guidelines in general practice: observational study. British Medical Journal. 1998; 317: 858-861.

[29] Fonarow GC, Abraham WT, Albert NM, Gattis Stough W, Gheorghiade M, Greenberg $\mathrm{BH}$, et al; Influence of a performanceimprovement initiative on quality of care for hospitalized patients with heart failure: Results of the organized program to initiate lifesaving treatment in hospitalized patients heart failure (OPTIMIZE-HF). Archives of Internal Medicine.2007; 167: 1493-1502.

[30] Craig P, Dieppe P, Macintyre S, Michie S, Nazareth I, Petticrew 
M, et al. Developing and evaluating complex interventions: the new Medical Research Council guidance. British Medical Journal. 2008; 337: a1655.

[31] Craig P, Dieppe P, MacItyre S, Mitchie S, Nazareth I, Petticrew M. Developing and evaluating complex interventions: the new Medical Research Council Guidance. British Medical Journal. 2008; 337: a1655.

[32] Sheridan DJ, Julian DG. Achievements and limitations of evidence-based medicine. Journal of the American College of Cardiology. 2016; 68: 204-213.

[33] Evidence-Based Medicine Working Group. Evidence-based medicine: a new approach to teaching the practice of medicine. Journal of the American Medical Association. 1992; 268: 2420 2425.

[34] Loughlin M, Bluhm R, Buetow S, Upshur REG, Goldenberg MJ, Borgerson $\mathrm{K}$, et al. Reason and value: making reasoning fit for practice. Journal of Evaluation in Clinical Practice. 2012; 18: 929-937.

[35] Ghali JK, Massie BM, Mann DL, Rich MW. Heart failure guidelines, performance measures, and the practice of medicine. Journal of the American College of Cardiology. 2010; 56: $2077-$ 2080.

[36] Bonow RO, Bennett S, Casey DE, Ganiats TG, Hlatky MA, konstam ma, et al. ACC/AHA clinical performance measures for adults with chronic heart failure. Journal of the American College of Cardiology. 2005; 46: 1144-1178.

[37] Kersten JR. A recipe for perioperative cardioprotection. Circulation. 2012; 126: 2671-2673.

[38] Lionetti V, Barile L. Perioperative cardioprotection: back to bedside. Minerva Anestesiol. 2020; 86: 445-454.

[39] Agrimi J, Baroni C, Anakor E, Lionetti V. Perioperative HeartBrain Axis Protection in Obese Surgical Patients: The Nutrigenomic Approach. Current Medicinal Chemistry. 2020; 27: 258281

[40] McAlister FA, van Diepen S, Padwal RS, Johnson JA, Majumdar SR. How evidence-based are the recommendations in evidencebased guidelines? PLoS Medicine. 2007; 4: e250.

[41] Guyatt GH, Oxman AD, Vist GE, Kunz R, Falck-Ytter Y, Alonso-Coello P, Schunemann HJ. GRADE: an emerging consensus on rating quality of evidence and strength or recommendations. British Medical Journal. 2008; 336: 924-926.
[42] Smith SC. Risk-Reduction therapy: the challenge to change. Circulation. 1996; 93: 2205-2211.

[43] DeMaria AN. Clinical trials and clinical judgment. Journal of the American College of Cardiology. 2008; 51: 1120-1122.

[44] Clark AM, Savard LA, Thompson DR. What is the strength of evidence for heart failure disease-management programs? Journal of the American College of Cardiology. 2009; 54: 397-401.

[45] Jaarsma T, van der Wal MH, Lesman-Leegte I, Luttik ML, Hogenhuis J, Veeger NJ, et al. Effect of moderate or intensive disease management program on outcome in patients with heart failure coordinating study evaluating outcomes of advising and counseling in heart failure $(\mathrm{COACH})$. Archives of Internal Medicine. 2008;168: 316-324.

[46] Nucifora G, Albanese MC, De Biaggio P, Caliandro D, Gregori $\mathrm{D}$, Goss $\mathrm{P}$, et al. Lack of improvement of clinical outcomes by a low-cost, hospital-based heart failure management program. Journal of Cardiovascular Medicine. 2006; 7: 614-622.

[47] Smith B, Forkner E, Zaslow B, Krasuski RA, Stajduhar K, Kwan $\mathrm{M}$, et al. Disease management produces limited quality-of-life improvements in patients with congestive heart failure: evidence from a randomized trial in community dwelling patients. The American Journal of Managed Care. 2005; 11: 701-713.

[48] Nguyen V, Ducharme A, White M, Racine N, O'Meara E, Zhang $\mathrm{B}$, et al. Lack of long-term benefits of a 6-month heart failure disease management program. Journal of Cardiac Failure. 2007; 13: $287-293$

[49] Holland R, Brooksby I, Lenaghan E, Ashton K, Hay L, Smith $\mathrm{R}$, et al. Effectiveness of visits from community pharmacists for patients with heart failure: heartmed randomised controlled trial. British Medical Journal. 2007; 334: 1098.

[50] Ledwidge M, Ryan E, O'Loughlin C, Ryder M, Travers B, Kieran E, et al. Heart failure care in a hospital unit: a comparison of standard 3-month and extended 6-month programs. European Journal of Heart Failure. 2005; 7: 385-391.

[51] Boutron I, Moher D, Altman DG, Schulz KF, Ravaud P. Extending the CONSORT statement to randomized trials of nonpharmacologic treatment: explanation and elaboration. Annals of Internal Medicine. 2008; 148: 295.

[52] Campbell NC, Murray E, Darbyshire J, Emery J, Farmer A, Griffiths F, et al. Designing and evaluating complex interventions to improve health care. British Medical Journal. 2007; 334: 455459 . 